\title{
On catching on to idiomatic expressions
}

\author{
SAMUEL A. BOBROW and SUSAN M. BELL* \\ University of Washington, Seattle, Washington 98195
}

\begin{abstract}
These experiments concern the comprehension of idiomatic expressions. The hypothesis was that there are distinct idiomatic and literal modes of processing sentences. In two experiments, 414 undergraduates read a series of sentences containing either literal or idiomatic ambiguities and then a test which had both a literal and an idiomatic meaning. Ss recorded, which meaning they perceived first. Taken together, the experiments indicate that inducing a set to perceive idioms can increase the proportion of people seeing the idiomatic meaning of test sentence first and a set to perceive literal meanings can reduce this proportion compared to a no-set baseline. Since the procedures to induce set did not involve grammatical or semantic information relevant to comprehension of the test sentence, these results suggest the existence of distinct literal and idiomatic processing strategies.
\end{abstract}

The idiom is abundant in everyday speech, but rare as the subject of psychological research. Idioms appear in every language; indeed, Weinreich (1969) notes that a Russian phraseological dictionary lists more than 25,000 entries. In general, linguists have dealt with idiomaticity as a semiproductive pattern within a generative grammar (e.g., Katz \& Postal, 1964; Weinreich, 1969). The current study approaches the idiom from the point of view of information processing rather than linguistic analysis and is concerned with comprehension rather than production.

Weinreich (1969) defines an idiom as "a complex expression whose meaning cannot be derived from the meanings of its elements." Chafe (1970) has pointed out that many idiomatic expressions are ambiguous, with one interpretation (the literal meaning) deriving from the meanings of the words involved and the other (idiomatic meaning) following the Weinreich definition. Transformational grammarians have defined three types of ambiguity: lexical, surface structure, or underlying structure ambiguities (see Chomsky, 1965). Lexical ambiguity involves alternative dictionary meanings of a word (e.g., "The cold was bothering John"). Surface (or derived) structure ambiguity involves a change in the grouping together of adjacent words into phrases (e.g., "John knows how many people fail"). Underlying structure ambiguity involves a change in the essential relations between words (e.g., "The mayor told the police to stop drinking"). Note that idiomatic ambiguity is not described by any of the three definitions. That is, the idiom "John let the cat out of the bag" cannot be understood to mean "John told the secret" by simply changing the meaning of one word, by regrouping adjacent words into a new phrase structure, or by reinterpreting the relationships between some of the words. Instead, the idiomatic meaning seems to be understood by combining several words into a complex

\footnotetext{
*Requests for reprints should be sent to Susan M. Bell, Department of Psychology, University of Washington, Seattle, Washington 98195 .
}

"idiom word" and finding the meaning of the phrase by a search through a nental "idiom word" dictionary.

Discovery of the idiomatic meaning of an idiom seems to result from processing the idiom as a word. Such a processing strategy differs from that which has been suggested for literal expressions, wherein each word is perceived, its meanings discovered, and then mapped into a semantic whole such that the meanings of the individual words relate to produce a meaning for the sentence (Quillian, 1968). Empirical substantiation of this intuitive dichotomy of literal and idiomatic processing modes was the main purpose of the current investigations.

The design of the present study is a "set" paradigm, similar to that of Marshall (1965), Carey, Mehler, and Bever (1970), and MacKay (1970). These studies demonstrated that establishing a set to perceive a particular syntactic structure biases the meaning Ss will see when subsequently presented a literally ambiguous sentence. They presented a literally ambiguous sentence following several unambiguous sentences having the same grammatical structure as one of the meanings of the ambiguous sentence. The Ss were found to perceive the ambiguous sentence as having the set structure. For example, when sentences such as "They are unearthing diamonds" and "They are installing benches" preceded the sentence "They are visiting sailors," the S interpreted "They are visiting sailors" differently from when it was preceded by sentences of the grammatical structure of "They are incoming signals" or "They are emerging nations."

Similarly, it has been shown that priming the $\mathrm{S}$ with supplemental semantic information also influences which meaning of an ambiguous sentence will be perceived. In a dichotic listening experiment, Garrett (1970) presented a sentence such as "Baseball spectators were yelling" to the "unattended" ear simultaneously with ambiguous input such as "The fans were noisy that night" to the "attended" ear and found that Ss tended to understand the ambiguous sentence as having to do with people rather than with mechanical devices. Thus, 
both syntactic and semantic cues or sets have been shown to be effective in influencing which meaning of an ambiguous sentence will be perceived.

The logic of the current investigation revolves around the establishment of a processing set. i.e., biasing the $S$ to employ a literal processing strategy or to employ an idiomatic processing strategy to discover the meaning of an idiomatically ambiguous sentence. It seems clear that if a semantic cue such as "mystery" were given first, a S would be most likely to interpret "John let the cat out of the bag" as meaning "John told the secret." If, however, the $S$ can be programmed to find or avoid finding the idiomatic meaning of a sentence in the absence of specific semantic or grammatical cues, we claim this as evidence for separate idiomatic and literal modes of processing.

In the experiment, to induce a set for idiom processing, a $\mathbf{S}$ read four idiomatic expressions. A fifth sentence was ambiguous, having both idiomatic and literal meanings. To induce a set for literal processing, $S$ read four sentences with only literal meanings. The critical test sentence also had both literal and idiomatic meanings. The S's task was to find two meanings for each sentence and indicate which meaning was perceived first. The first sentence presented in the idiom set condition also had a literal meaning. The proportion of Ss perceiving the idiomatic meaning first was taken as the baseline or probability of idiom-first response, given no bias. The hypothesis was that a set for idiom processing would result in a higher-than-baseline probability of idiom-first responding for the fifth sentence, whereas a set for literal processing would lower this probability below baseline for the test sentence.

\section{EXPERIMENT I}

\section{Method}

\section{Subjects}

The Ss were 152 undergraduate students enrolled in two psychology courses at the University of Washington. Of these, half (76) served in the idiomatic set condition and half in the literal set condition. The students in each course served simultaneously in a group experiment. Assignment of Ss to condition was semirandom.

\section{Materials}

Each $S$ was given a test booklet consisting of five white pages covered and separated by colored sheets. On the first side of each white page appeared an ambiguous sentence, and on the reverse side were the two meanings of that sentence. For example, for the ambiguous sentence "Mary fed her dog biscuits," the two sentences on the reverse side of the page were "Mary gave biscuits to her dog" and "Mary gave dog biscuits to some woman." The order of appearance of the alternative meanings for each sentence was counterbalanced.

In the literal set, the first four sentences contained single literal ambiguities (e.g., "Mary fed her dog biscuits") and the last sentence contained one of four idiomatic ambiguities (e.g., "John gave Mary the slip"). The order of the literally ambiguous sentences preceding a given idiomatic sentence was counterbalanced over booklets. The other literal sentences were "John observed the wild Indian dance." "John saw the children run in the house." and "John and Mary know how many people fail." The four idiomatic sentences used as the test sentences in the literal set appeared as both set and test sentences in the idiomatic set. along with a filler idiom ("John smelled a rat") which always appeared on Page 3. Thus, the idiomatic set booklets contained tive idiomatically ambiguous sentences. the first four forming the set and the fifth being the test sentence within a given booklet. Other sentences were "John and Mary buried the hatchet," "John gave Mary the slip," "John let the cat out of the bag." and "John was in hot water."

\section{Procedure}

The two test sessions were held $2 \mathrm{~h}$ apart. The procedure was the same for each session. The Ss were instructed that, when told to begin. they were to read the ambiguous sentence appearing on the first white page of their booklet and to discover the two meanings. then to turn the page and mark the sentence corresponding to the meaning they perceived first. The Ss were told that if they could not see both meanings after about $10 \mathrm{sec}$, they should turn the page. mark the meaning they did see. and indicate that they saw only that meaning. The Ss continued in this manner through the five sentences in their booklets. The Ss were instructed to indicate their sex on the booklet cover.

\section{Results}

The proportion of Ss who reported first having seen the idiomatic meaning of Sentence 1 of the idiomatic set was calculated. This information corresponds to the "bias" of an ambiguous sentence as defined by MacKay (1970), i.e., the percentage of Ss who first see a given meaning. Since the interpretation of the sentence appearing first in a booklet could not have been influenced by experimental set, responses to Sentence 1 of the idiomatic set defined a baseline bias condition (no set condition). In the no set condition, 62 of the $76 \mathrm{Ss}$ saw the idiomatic meaning first, representing a proportion of "saw idiom first" responses of .82 .

Effects of the idiomatic set were evaluated on the basis of the meaning first seen for the fifth sentence in the booklet. In the idiomatic set condition, 64 of the 76 Ss saw the idiomatic meaning first, corresponding to a proportion of 84 .

Effects of the literal set were scored by meaning first seen for the final (idiomatic) sentence in the literal set booklet. In the literal set, 43 of the 76 Ss first saw the idiomatic meaning, the proportion of such responses being .57. Table 1 presents these proportions for each of the sentences.

A chi-square test for correlated data was used to compare the no set and idiomatic set conditions. There was no significant difference $\left(\chi^{2}=2.22\right)$. However, a chi-square test for independent data indicated that the no set and literal set conditions were significantly different $\left(\chi^{2}=11.4, p<.001\right)$. The "saw idiomatic first" proportion was significantly lower in the literal set condition.

The "saw idiom first" proportion for all four 
sentences in the no set, idiomatic set, and literal set conditions was analyzed. There were no significant differences among sentences within any one condition. There were also no differences between male and female response patterns.

\section{Discussion}

This experiment demonstrated that the "saw idiomatic first" proportion could be reduced below baseline levels by a set to process sentences in a literal mode. However, the set for idiom processing was not effective in raising the proportion. The baseline proportion $(.80)$ of the sentences used seemed very high, perhaps at ceiling levels.

\section{EXPERIMENT II}

The main purpose of Experiment II was to replicate the first experiment with sentences that had lower "saw idiomatic first" proportions. If the failure to find an increase in "saw idiomatic first" proportions following the idiomatic set was due to ceiling baseline proportions, then the idiomatic set should be effective for low baseline sentences. A secondary purpose of Experiment II was to test the notion that idiom processing is most closely related to the processing involved in discovering lexical ambiguities. To this end, there were two literal set conditions, differing by the kind of literal ambiguity of the set-inducing sentences. The two kinds of literal ambiguity were surface structure and lexical. The process of finding a meaning for a sentence with a lexical ambiguity must involve deciding on one meaning of the lexical ambiguity. Similarly, if an idiom is regarded as a compound lexical unit, the process of finding one meaning for an idiomatic sentence would also involve deciding on one meaning for a lexical unit. The process of finding a meaning for a sentence with a surface structure ambiguity would seem to involve deciding on how to combine meanings of lexical units. Hence, it would seem that idiomatic ambiguity might be more similar to lexical ambiguity than surface structure ambiguities. The hypothesis was that the "saw idiomatic first" proportion would be higher following lexically, rather than following surface structurally, ambiguous sentences.

\section{Method}

The method employed in Experiment II was the same as that reported for Experiment I, except as noted below.

There were 262 undergraduates enrolled in four courses who served as Ss. Of these, 93 served in the idiomatic set condition. The literal set was expanded to form two conditions, differentiated by the type of literal ambiguity employed. In the surface structure condition, the first four sentences contained surface structure ambiguities and there were $93 \mathrm{Ss}$. In the lexical set condition, the four set sentences contained lexical ambiguities and $76 \mathrm{~S}$ s participated.
Table 1

Proportion of Individuals Who Reported Seeing the Idiomatic Meaning of the Sentence First in the Three Conditions of Experiment I

\begin{tabular}{lccc} 
Sentence & $\begin{array}{c}\text { Lite- } \\
\text { No } \\
\text { ral } \\
\text { Set }\end{array}$ & $\begin{array}{c}\text { Idio- } \\
\text { matic } \\
\text { Set }\end{array}$ \\
\hline John let the cat out of the bag. & .79 & .53 & .79 \\
John was in hot water. & .84 & .63 & .84 \\
John and Mary buried the hatchet. & .84 & .53 & .74 \\
John gave Mary the slip. & .79 & .59 & .95 \\
\hline
\end{tabular}

\section{Materials}

Each test booklet contained six ambiguous sentences, the first five forming the experimental set and the sixth being an idiomatic test sentence. An attempt was made to use idiomatic test sentences which might have a low no set bias on their idiomatic meanings, i.e., sentences for which the proportion of Ss first seeing the idiomatic meaning in the no set condition would be low. Therefore, in the idiomatic set booklets, the first and last sentences were always two of these potentially low-bias idioms, with four high-bias idioms from Experiment I appearing as Sentences 2-5 and forming the experimental set. The sentences which contained potentially low-bias idioms were "John stabbed Mary in the back," "John had sticky fingers," "John was climbing the walls," and "John made a big splash." In the lexical set and surface set booklets, the first five sentences contained the appropriate type of literal ambiguities, the sixth sentence being the test, low-bias idiom. In the lexical set, these sentences were "The cold was bothering John," "John wore a light suit," "John bought the plant," "The fans were noisy," and "John put gasoline into the tank." The surface set sentences were "John and Mary know how many people fail," "John and Mary talked about the problem with the president," and "John told Mary to go without any hesitation."

\section{Procedure}

The experiment was conducted with four groups over a period of 2 days, again with Ss assigned semirandomly to condition.

\section{Results}

An examination of responses in the no set condition revealed that only three of the four test sentences actually had a low no set bias on their idiomatic meanings. "Saw idiom first" responses to the fourth sentence represented a proportion of .74. For this reason, responses to this sentence were eliminated from the data analysis. The proportion of Ss first seeing the idiomatic meaning of the three remaining sentences in the no set was .22 .

In the idiomatic set, 35 of the $70 \mathrm{Ss}(.50)$ reported first seeing the idiomatic meaning. The proportion of "saw idiom first" responses in the lexical set was .26, 16 of the 57 responses being in this category. This proportion in the surface structure set was .19 (14 of the 72 responses). Table 2 presents these proportions for each sentence.

A chi-square test for correlated data was used to compare the no set and idiomatic set conditions. The "saw idiomatic first" proportion was significantly higher 
Table 2

Proportion of Individuals Who Reported Seeing the Idiomatic Meaning of the Sentence First in the Four Conditions of Experiment II

\begin{tabular}{|c|c|c|c|c|}
\hline Sentence & $\begin{array}{l}\text { No } \\
\text { Set }\end{array}$ & $\begin{array}{c}\text { Literal } \\
\text { Lexi- } \\
\text { cal } \\
\text { Set }\end{array}$ & $\begin{array}{l}\text { Sur- } \\
\text { face } \\
\text { Set }\end{array}$ & $\begin{array}{c}\text { Idio- } \\
\text { matic } \\
\text { Set }\end{array}$ \\
\hline John had sticky fingers. & .29 & .44 & .12 & .42 \\
\hline John stabbed Mary in the back. & .19 & .22 & .12 & .52 \\
\hline John was climbing the walls. & .74 & .90 & .81 & .82 \\
\hline John made a big splash. & .21 & .19 & .35 & .57 \\
\hline
\end{tabular}

in the idiomatic set condition $\left(\chi^{2}=18.9, p<.001\right)$. The same test with data from all of the sentences also resulted in a highly significant chi square.

A chi-square test for independent data indicated that the "saw idiomatic first" proportions in the no set, lexical set, and surface structure set were not significantly different $\left(\chi^{2}=1.46\right)$. This chi square was not significant when the data from all four sentences were used in the test.

\section{Discussion}

In Experiment I, the literal set condition significantly decreased the proportion of Ss first seeing the idiomatic meaning of the test sentence, while the idiomatic set condition did not significantly alter the bias of the idiomatic sentences used.

Since the baseline bias of the no set sentences used in Experiment I was approximately .80, the failure of the idiomatic set condition to produce significant results might have been due to a ceiling effect. That is, it may simply have been the case that these idioms were already so highly biased that increasing the bias would be extremely difficult.

For this reason, Experiment II was conducted using idiomatic sentences with a low no set bias. These sentences proved to each have a no set bias approximating .20 (with the exception of the sentence eliminated from the analysis). By the ceiling effect argument, we would expect that it would be difficult to reduce the bias of low-bias sentences. The idiomatic set significantly increased the proportion of Ss seeing the idiomatic meaning first, but neither literal condition, surface structure set, or lexical set was effective in lowering the proportion of such responses.

Taking the two experiments together, exposure of high-idiomatic bias idioms to the literal set condition did decrease the bias to see the idiomatic meaning first, and in Experiment II, exposure of low-idiomatic bias idioms to the idiomatic set condition increased this bias. The set treatments did not provide additional semantic information which would favor one of the interpretations of the idiomatic test sentences (all sentences employed were quite diverse semantically), nor did the set conditions provide consistent grammatical cues (there was no consistent syntactic structure involved). The grammatical (syntactic) and semantic components of the written sentence fully describe its information content, and since the set conditions employed did not rely upon grammatical or semantic cues, the observed effects can only be ascribed to differences in mode of processing. In effect, the Ss were being told what processing strategy to employ-idiomatic or literal. The fact that the use of the two strategies resulted in differential perception of ambiguous sentences is evidence for the existence of distinct processing modes.

In Experiment II, the possibility that idiomatic ambiguity is more closely related to lexical ambiguity was also studied by forming two literal conditions, the lexical set and the surface structure set, and comparing their effects upon perception of a subsequent idiomatic ambiguity. The prediction was that the lexical set would act like the idiomatic set, if, indeed, there is a close relation between the two types of ambiguity. The lexical set and the surface structure set did not produce significantly different effects in Experiment II. Nonetheless, the trend in the predicted direction shown in the data suggests that further research along this line may be in order.

The current study evidenced the existence of distinct processing modes for idiomatic and literal ambiguities. Further research is needed to define the variables that determine the choice of processing modality and the precise differences in the two modes of processing.

\section{REFERENCES}

Carey, P., Bever, T., \& Mehler, J. When do we compute all the interpretations of an ambiguous sentence. In W. Levelt and G. Flores d'Arcais (Eds.), Advances in psycholinguistics. Amsterdam: North-Holland, 1970.

Chafe, W. Meaning and the structure of language. Chicago: University of Chicago, 1970.

Chomsky, N. Aspects of the theory of syntax. Cambridge: M.I.T. Press, 1965.

Garrett, M. Does ambiguity complicate the perception of sentences? In W. Levelt and G. Flores d Arcais (Eds.), Advances in psycholinguistics. Amsterdam: North-Holland, 1970.

Katz, J., \& Postal, P. An integrated theory of linguistic descriptions. Cambridge: M.I.T. Press, 1964.

MacKay, D. Mental diplopia: Towards a model of speech perception at the semantic level. In W. Levelt and G. Flores d'Arcais (Eds.), Advances in psycholinguistics. Amsterdam: North-Holland, 1970.

Marshall, J. Syntactic analysis as a part of understanding. Bulletin of the British Psychological Society, 1965, 18, 28.

Quillian, R. Semantic memory. In M. Minsky (Ed.). Semantic information processing. Cambridge: M.I.T. Press. 1968.

Weinreich, U. Problems in the analysis of idioms. In J. Puhvel (Ed.), Substance and structure of language. Berkeley: University of California, 1969.

(Received for publication December 22, 1972; revision received March 5, 1973.) 